\section{Serum Biomarkers of COPD}

\section{To the Editor:}

Ambade et $\mathrm{al}^{1}$ conducted an observational study to evaluate the potential serum biomarkers in subjects with COPD. The authors assessed superoxide dismutase-3, glutathione peroxidase, catalase, ceruloplasmin ferroxidase activity, C-reactive protein, and surfactant protein $\mathrm{D}$ in the serum of subjects with COPD and reported that ceruloplasmin ferroxidase activity and glutathione peroxidase are the most likely potential serum biomarkers in COPD. The study involved 96 stable COPD subjects in the study group and 96 subjects in the control group. In both groups, the number of males was higher than the number of females (male/female ratio of 73:23). Prior studies have reported that plasma glutathione peroxidase levels are significantly elevated in the plasma of females compared to males. ${ }^{2}$ Thus, the glutathione peroxidase activity measured by the Ambade et $\mathrm{al}^{1}$ is possibly attributable to the female population. It would have been interesting to see the glutathione peroxidase analysis of males and females separately for better understanding of glutathione peroxidase activity.

Prior studies have reported that there is poor correlation of respiratory symptoms and lung function data in subjects with asthma. ${ }^{3}$ Ambade et $\mathrm{al}^{1}$ did not conduct any lung function tests on the control subjects because none of the control subjects had exhibited any respiratory symptoms, indicating incomplete subject information. Thus, the authors could have possibly measured the lung function parameters of controls as well for better understanding of the subject population involved in the study.

Studies have also reported that smokers tend to have higher C-reactive protein levels compared with nonsmokers. ${ }^{4}$ It would have also been interesting to see a comparison within the healthy and COPD subgroups (ie, healthy nonsmokers versus healthy smokers and smokers in the COPD group versus nonsmokers in the COPD group).

Last, the authors could have possibly correlated the ceruloplasmin ferroxidase activity and glutathione peroxidase data with lung function. This would have made the results easier to comprehend to better understand the disease pathogenesis and support the sensitivity of ceruloplasmin ferroxidase activ- ity and glutathione peroxidase as effective biomarkers of COPD.

Baishakhi Ghosh MSc

Chest Research Foundation

Pune, Maharashtra, India

Faculty of Health and Biomedical Science Symbiosis International University

Pune, Maharashtra, India

Ms Ghosh has disclosed no conflicts of interest.

DOI: $10.4187 /$ respcare.04592

\section{REFERENCES}

1. Ambade VN, Sontakke AN, Barthwal MS, Tyagi R, Basannar DR. Diagnostic utility of biomarkers in COPD. Respir Care 2015; 60(12):1729-1742.

2. Rush JWE, Sandiford SD. Plasma glutathione peroxidase in healthy young adults: influence of gender and physical activity. Clin Biochem 2003;36(5):345-351.

3. Teeter JG, Bleecker ER. Relationship between airway obstruction and respiratory symptoms in adult asthmatics. Chest 1998; 113(2):272-277

4. Ohsawa M, Okayama A, Nakamura M, Onoda $\mathrm{T}$, Kato $\mathrm{K}$, Itai $\mathrm{K}$ et al. C-reactive protein levels are elevated in smokers but unrelated to the number of cigarettes and are decreased by long-term smoking cessation in male smokers. Prev Med 2005;41(2): 651-656.

\section{Serum Biomarkers of COPD—Reply}

In reply:

I appreciate the efforts taken by the author of the letter from Chest Research Foundation, Pune, who has responded to our article. ${ }^{1}$ First of all, I could not understand the meaning of the sentence "Prior studies have reported that plasma glutathione peroxidase levels were significantly elevated in plasma than males." I believe that the author of the letter wants to reflect higher serum glutathione peroxidase levels in females as compared with males. Females showing higher serum glutathione peroxidase activity $(256.4 \pm 10.4 \mathrm{U} / \mathrm{L}$ vs $222.8 \pm 15.6 \mathrm{U} / \mathrm{L}$ $P<.05)$ than males have been reported by Rush and Sandiford. ${ }^{2}$ However, the possibility of attributing the higher glutathione peroxidase activity measured in our study ${ }^{1}$ is attributable to a predominantly female rather than male population, as suggested by the author, may not be relevant. What we have highlighted in this study is a dif- ference in the levels of glutathione peroxidase in the control group $(50.95 \pm 15.30$ $\mathrm{U} / \mathrm{L})$ as compared with the COPD group $(89.73 \pm 27.84 \mathrm{U} / \mathrm{L})$. In this study, even if both the COPD group and control group had consisted of equal numbers of males and females, the mean difference between the COPD group and control group would have been the same and led to the same conclusions. Taking more females into the control group and COPD group to match the number of males might have slightly increased the glutathione peroxidase levels in both groups, but the mean difference probably would still remain almost the same. This article was not aimed at establishing the normal reference range of glutathione peroxidase, yet I agree with the author that equal numbers of males and females in the study groups would have been better.

I agree with the comment of the author that prior studies have reported poor correlation of respiratory symptoms and lung function data in subjects with asthma. The same results may be found for COPD patients. Since none of the control subjects in this study had respiratory problems or lung diseases or were receiving inhaled bronchodilators or corticosteroids, spirometry for lung function data in the normal group was not conducted. This was in accordance with the guidelines of the American College of Physicians, American College of Chest Physicians, American Thoracic Society, and European Respiratory Society. ${ }^{3}$

The author of the letter has pointed out that these studies reported that smokers have higher C-reactive protein levels compared with nonsmokers and suggested that it would have been interesting to see a comparison within the healthy and COPD subgroups (ie, healthy nonsmokers vs healthy smokers and smokers in the COPD group vs nonsmokers in the COPD group). I fully agree with the author that smokers have higher C-reactive protein levels. I further want to inform the author that this had already been done, and all of this information was provided while replying to a reviewer's comments during publication of the article, since the same query was expressed by one of the reviewers. However, the comparison is given once again along with glutathione peroxidase levels, as desired by the author, in Table 1.

I have the same opinion as that of the author regarding the correlation of ferroxidase activity of ceruloplasmin and glutathi- 